\title{
Physical exercise and internet-based cognitive-behavioural therapy in the treatment of depression: randomised controlled trial
}

Mats Hallgren, Martin Kraepelien, Agneta Öjehagen, Nils Lindefors, Zangin Zeebari, Viktor Kaldo and Yvonne Forsell

\section{Background}

Depression is common and tends to be recurrent. Alternative treatments are needed that are non-stigmatising, accessible and can be prescribed by general medical practitioners.

\section{Aims}

To compare the effectiveness of three interventions for depression: physical exercise, internet-based cognitivebehavioural therapy (ICBT) and treatment as usual (TAU). A secondary aim was to assess changes in self-rated work capacity.

\section{Method}

A total of 946 patients diagnosed with mild to moderate depression were recruited through primary healthcare centres across Sweden and randomly assigned to one of three 12-week interventions (trail registry: KCTR study ID: KT20110063). Patients were reassessed at 3 months (response rate $78 \%$ ).

\section{Results}

Patients in the exercise and ICBT groups reported larger improvements in depressive symptoms compared with TAU Work capacity improved over time in all three groups (no significant differences).

\section{Conclusions}

Exercise and ICBT were more effective than TAU by a general medical practitioner, and both represent promising non-stigmatising treatment alternatives for patients with mild to moderate depression.

\section{Declaration of interest}

None.

\section{Copyright and usage}

(c) The Royal College of Psychiatrists 2015.
Depression is a common psychiatric disorder and a major contributor to mortality and morbidity worldwide. Over the past decade in Sweden, work absence because of mental ill health has risen markedly and depression is a major factor. ${ }^{1}$ Substantial societal costs are associated with the disorder, which affects up to $15 \%$ of the population at any one time and tends to be recurrent. ${ }^{2}$ There are compelling reasons to investigate alternative treatments for depression. Although effective treatments exist, most people with the disorder never seek professional help. Among those that do, only half appear to benefit. ${ }^{3}$ Adherence with medication is often poor and waiting times for cognitive-behavioural therapy (CBT) can be lengthy, resulting in more entrenched symptoms and a worse long-term prognosis. As general medical practitioners are frequently the main care providers for depression, treatment options that are non-stigmatising, have few side-effects and can readily be prescribed in community healthcare settings are needed.

Prescribed physical exercise has been proposed by many as an effective alternative treatment for depression and meta-analysis has demonstrated effect sizes ranging from -0.80 to $-1.1{ }^{4} \mathrm{~A}$ recent Cochrane review of 32 randomised controlled trials (RCTs) concluded that exercise is moderately more effective than a control intervention for reducing the symptoms of depression. ${ }^{5}$ Several models have been proposed to explain the benefits of regular exercise on mood disorders. They include changes in core body temperature, increased serotonin synthesis and hippocampal cell proliferation $^{6,7}$ and reduced levels of proinflammatory cytokines. ${ }^{8}$ Psychological theories suggest that distraction, mastery and improved self-efficacy also play a role. ${ }^{9}$ Internet-based interventions to promote health have expanded greatly over the past decade. ${ }^{10}$ Such interventions have the advantage of being able to reach individuals without access to conventional treatment. This includes people living in rural or remote areas and individuals with mobility issues. Like exercise, internet-based therapy may also help to reduce patient waiting times for treatment. CBT is considered a first-line treatment for depression with strong empirical support. ${ }^{11} \mathrm{CBT}$ can be made available via the internet in the form of guided self-help. Internet-based CBT (ICBT) engages patients in structured programmes of care through online modules with limited online message support from a qualified therapist. Several RCTs have assessed the effectiveness of ICBT on depression. ${ }^{12,13} \mathrm{~A}$ recent review concludes that internet-based psychological treatments can be equally effective in treating mild to moderate depression as face-to-face CBT. ${ }^{14}$

Physical exercise for depression is currently recommended in Sweden and ICBT-interventions continue to grow. However, it remains unclear which treatment option is most effective and how each compares with conventional treatments routinely prescribed in clinical practice. The aim of the present study, therefore, was to compare the effectiveness of regular physical exercise and ICBT to standard treatments for mild to moderate depression in a population of Swedish adults. The primary outcome of interest was self-rated depression. As mood disorders frequently have an impact on work performance, a secondary aim was to assess change in self-rated work capacity. In this paper, we present results from assessments taken at baseline (pre-treatment) and 3 months (post-treatment). The study is registered with the registry of clinical trials in Stockholm county (KCTR study ID: KT20110063).

\section{Method}

\section{Study design}

We conducted a RCT to assess the short-term effectiveness of three treatments for depression. As our primary focus was on the 
relative effectiveness of exercise and ICBT compared with treatment as usual (TAU), the latter group was considered a control equivalent. Each intervention lasted 12 weeks with assessments of depression and self-rated work capacity taken at baseline (pre-treatment) and post-treatment, 3 months later. The participants represent a cross-section of patients currently treated for depression in clinical practice because in addition to the three 12-week interventions, one-third of all patients were taking antidepressants (primarily selective serotonin reuptake inhibitors (SSRIs)) prior to and during the trial. An ethics committee at the Karolinska Institute approved the study (Dnr 2010/1779-31/4).

\section{Patient recruitment}

Patients were recruited via primary care facilities located in six county councils in Sweden (Stockholm, Skåne, Västra Götaland, Kronoberg, Blekinge and Västmanland). The selection of regions was deliberate and helped ensure that the sample included participants from different regions varying in population size and composition. Patients aged 18 years and over who scored $>9$ on the Patient Health Questionnaire (PHQ-9) ${ }^{15}$ were invited to participate in the trial. Recruitment began in February 2011 and the last participants ended their treatment in March 2013. Exclusion criteria were: age ( $<18$ years), severe somatic illness, a primary alcohol or drug use disorder, or a psychiatric diagnosis that required specialist treatment (such as psychosis). Following an initial consultation with their primary healthcare provider, suitable patients were referred to a research nurse who administered the baseline questionnaires and confirmed eligibility by conducting a thorough patient interview that included a psychiatric diagnostic assessment using standardised tools (described below). Before the study began, the research nurses were trained by a psychiatrist to interview patients and administer the questionnaires in a consistent manner. After obtaining informed consent, patients were randomised to one of the three treatment conditions by an independent clinical research organisation; the Karolinska Trial Alliance, where individual patients were treated as the 'unit' of randomisation. The blocks were unknown to the researchers, and a computer generated the group allocation. This procedure ensured that researchers responsible for the baseline and post-treatment assessments were masked to the patient's group allocation. Data on the number of patients who were invited to participate in the study but declined is unknown. It was not feasible for the primary care centres involved in the study to collect these data. In total, 946 patients met the inclusion criteria for the trial, agreed to participate and were included in the baseline analyses.

\section{Participant characteristics}

Baseline characteristics of the study sample are shown in Table 1. Most of the participants were women (73\%) with a mean age of 43 years (s.d. 12, range 18-71) and 78\% of the sample was employed or studying at baseline. Unemployment rates at baseline among men and women were $27 \%$ and $20 \%$, respectively, approximately three times higher than the national average for 2011. ${ }^{16}$ Based on the Mini-International Neuropsychiatric Interview (MINI), ${ }^{17}$ concurrent depressive and anxiety disorders were most common (67\%), however, some participants, exclusively, had either depression $(8 \%)$ or an anxiety disorder (20\%). Table 1 shows the primary diagnoses of all participants, based on the MINI assessment. A total of $68 \%$ of participants reported moderate to severe physical pain and believed that their daily activities were adversely affected by their health condition. Daily tobacco use was high (28\% of men, $18 \%$ of women) and the proportion of hazardous alcohol users exceeded the national average. ${ }^{18}$ As noted, one-third of all participants in the trial were taking antidepressants during the 12 -week intervention. The percentage within each group at baseline was: $31 \%$ in the physical exercise and ICBT groups, respectively, and $24 \%$ in the TAU group. The proportions were not significantly different $(P>0.07)$. The use of other medications (such as contraceptives, statins, thyroid drugs) during the trial was minimal.

\section{Post-treatment assessment}

All patients were contacted 3 months after commencing treatment by a research nurse (masked to group allocation) to arrange a post-treatment interview. In total $69 \%$ of the interviews were conducted at the patient's health clinic; $31 \%$ of the interviews were made by telephone. A short version of the baseline questionnaire was completed at follow-up (the MINI and AUDIT ${ }^{19}$ were not included), along with a brief exit survey to assess patient satisfaction with the trial. Patients who did not complete a post-treatment questionnaire were contacted by telephone on two separate occasions with a reminder. Those who did not respond to the second reminder were offered the possibility to complete the survey online $(n=17)$ or a short version of the survey by telephone $(n=38)$. The flow of patients through the trial is summarised in Fig. 1.

\section{Measures}

\section{Screening}

We used the PHQ-9 and the MINI. The PHQ-9 assesses the presence of nine key criteria for depression during the past 2 weeks

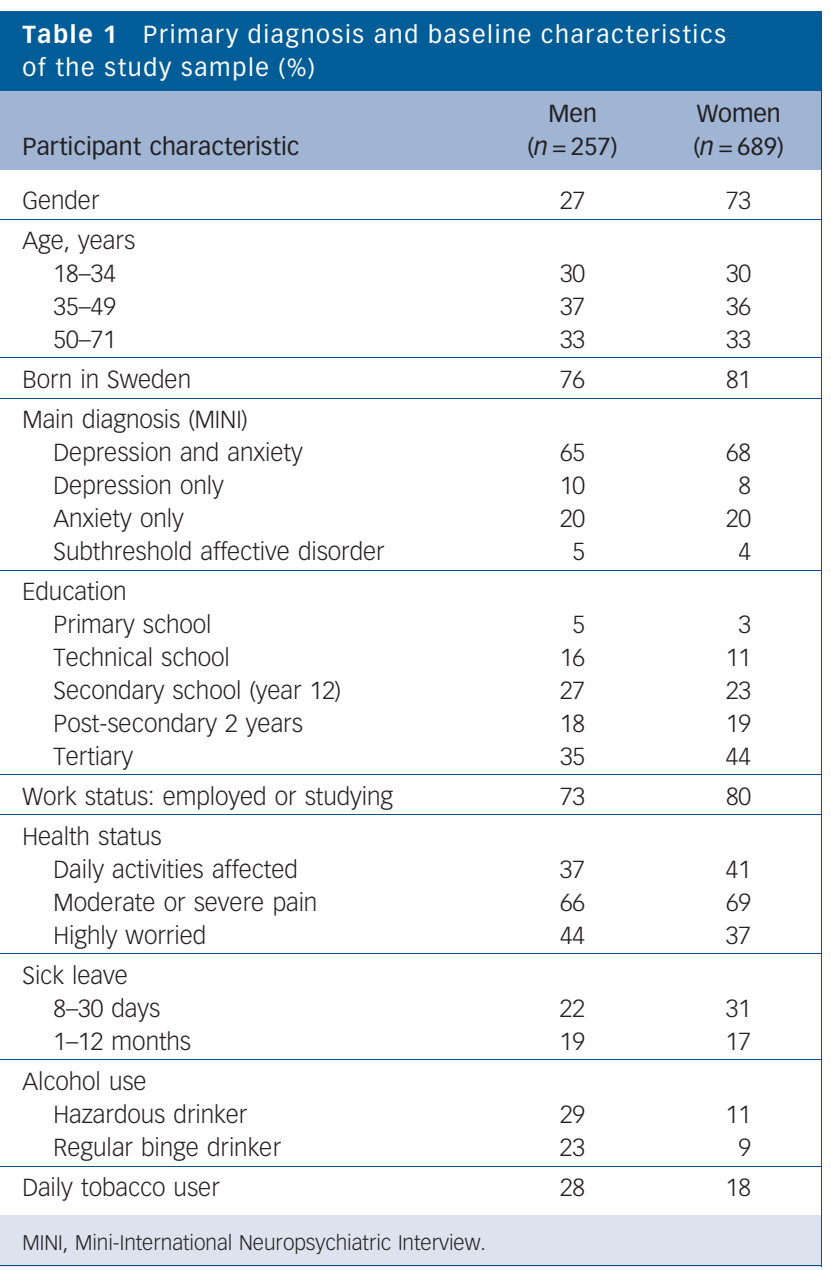




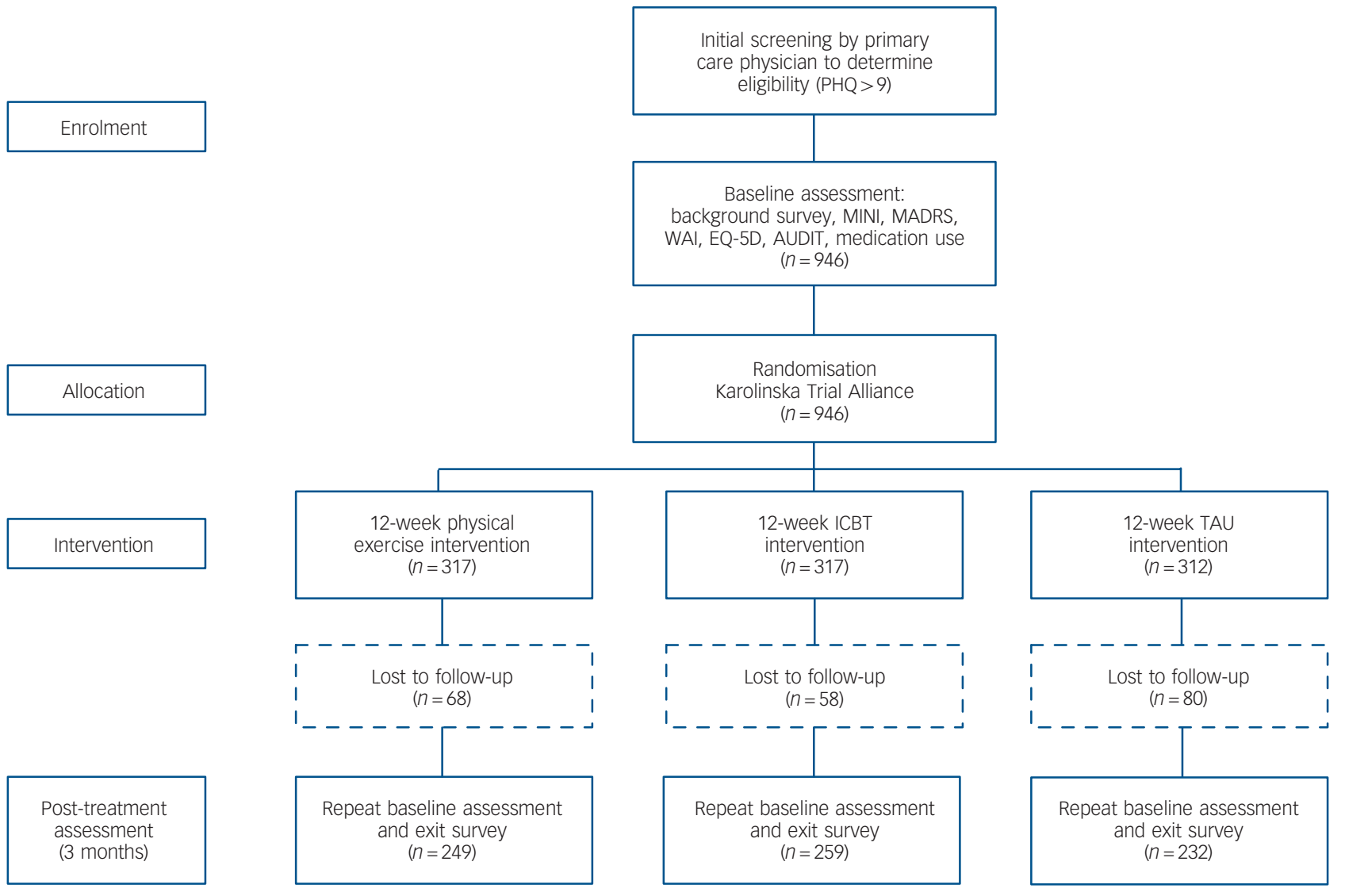

\section{Fig. 1 Flow chart of participants' progress}

PHQ-9, Patient Health Questionnaire; MINI, Mini-International Neuropsychiatric Interview; MADRS, Montgomery-Åsberg Depression Rating Scale; WAI, Work Ability Index; AUDIT, Alcohol Use Disorders Identification Test; ICBT, internet-based cognitive-behavioural therapy; TAU, treatment as usual.

(such as pleasure doing things, feeling down, difficulty sleeping). ${ }^{15}$ Patients rate the frequency of each symptom on a scale between 0 (not at all) to 3 (nearly every day). Total scores range from 0 to 27 . The PHQ-9 is a valid instrument for diagnosing depressive disorders and scores above 9 have a reported sensitivity and specificity of $88 \%$ for major depression. ${ }^{15}$ The $\mathrm{MINI}^{17}$ is a short, structured clinical interview used to diagnose psychiatric disorders based on the DSM-IV. ${ }^{20}$ The MINI was used primarily as a screening instrument to exclude patients who did not meet the study inclusion criteria but also to diagnose concomitant anxiety disorders. The complete MINI was performed.

\section{Baseline assessment}

The baseline assessment included the following. The EQ-5D was used to assess patient's overall health status. ${ }^{21}$ We report three key dimensions from the scale: main life activities, pain/discomfort and anxiety/depression. Each dimension is scored on a three-item scale indicating no problems, some problems or extreme problems. The Alcohol Use Disorders Identification Test (AUDIT) was used to assess the proportion of hazardous alcohol users and binge drinkers in the study. ${ }^{19}$ We used the recommended cut-off scores of 8 for men and 6 for women to define hazardous drinking. ${ }^{22}$ Binge drinking refers to the consumption of six or more standard drinks (or about $12 \mathrm{~g}$ of alcohol) on a single occasion. Participants who indicated binge drinking 'every month', 'every week' or 'daily', were coded as 'regular binge drinkers'. Those indicating 'never' or 'less than once per month' were excluded from this definition. Tobacco use was assessed with a single question: 'Do you smoke or use 'snus' daily?' with two response alternatives, yes or no (snus is a smokeless tobacco product). A questionnaire was developed by the research group to record the type of antidepressant medication used during the past 2 weeks. It was completed by a research nurse at baseline (pre-treatment) and 3 months (post-treatment).

\section{Primary and secondary outcomes}

The primary outcome was the severity of depression, assessed using the Montgomery-Åsberg Depression Rating Scale (MADRS); ${ }^{23}$ a 10 -item scale designed to be sensitive to changes in depressive symptoms following treatment. Higher scores indicate more severe depression; each item yields a score ranging from 0 to 6 . Ten symptoms are rated: apparent sadness, reported sadness, inner tension, reduced sleep, reduced appetite, concentration difficulties, lassitude, inability to feel, pessimistic and suicidal thoughts. Total scores range between 0 and 60 .

Our secondary outcome was self-rated work capacity. This was scored on a single 10-item Likert scale ranging from 0 (unable to work) to 10 (highest possible work capacity). The item was derived from the Work Ability Index (WAI) and is strongly correlated with the complete 24 -item WAI $(r=0.87) .^{24}$

\section{Interventions}

\section{Physical exercise}

Patients in the physical exercise group were randomised to one of three conditions: 'light exercise', which consisted of yoga classes (or similar) with a focus on gentle stretching and controlled breathing; 'moderate exercise', an intermediate-level aerobics class; and 'vigorous exercise', a higher intensity 
aerobics/strength-training and balance class. All exercise sessions were 60 min duration and most included between 5 and 20 participants. Patients were requested to participate in the exercise classes three times per week for 12 weeks. The exercise sessions were undertaken at 'Friskis och Svettis' a modern fitness centre with multiple locations throughout Sweden. As an incentive to increase adherence, all patients were provided with free gym memberships for 12 weeks. Adherence was monitored through weekly face-to-face meetings with a qualified personal trainer and patients who failed to attend this meeting were contacted by the trainer with a telephone call and encouraged to continue the exercise intervention. Reminder text messages were sent to patients who could not initially be contacted by telephone. Patients completed on average one exercise session per week; that is, 12 out of the recommended 36 sessions - an adherence rate of $33 \%$. There were no gender differences in exercise participation rates. As our main interest was the overall effect of the physical exercise intervention on depression data from the three intensity groups were analysed together. A forthcoming paper will report the effects of exercise intensity on treatment outcomes.

\section{ICBT}

Patients randomised to the ICBT condition received treatment through a secure website operated via the county council. Treatment involved the patient working through a self-help manual available online in the form of modules. The manual was mostly text based, but also included images and sound clips. Before commencing the treatment, all ICBT participants received a short telephone call from their assigned clinician (a psychologist) who explained the process. Initially, several online forms were completed to identify patient-specific mental health concerns and work-related problems. The basic modules completed during the first few weeks address problems related to depressive symptoms in general, such as inactivity and avoidance behaviours. Subsequent modules were patient specific and targeted different comorbid symptoms often seen in depression, such as worry, panic attacks, social anxiety, stress, insomnia and pain. Other patient-specific modules focused on problems related to the workplace and motivated the patient to return to work or find a new job. Throughout treatment, patient responses were monitored by the assigned clinician on a weekly basis so that high-risk individuals could receive additional help if necessary. Patients who did not use the service for 1 week or more were prompted by their clinician to continue the treatment (unless they had chosen to withdraw). Patients were able to contact a clinician directly or request additional support if needed. As with the physical exercise intervention, ICBT was offered free of charge. During the 3-month trial, patients logged into the website on average four times per week, sent 18 messages to their assigned therapist, received 17 messages and accessed 7.8 (s.d.=5) online modules out of an expected 13 (an adherence rate of 60\%). The average frequency of ICBT usage was higher among women across all measures (for example, women accessed the service 52 times over 12 weeks, compared with 40 times among men).

\section{TAU}

One-third of the participants received standard treatment for depression administered by their primary care physician who was responsible for determining the type of treatment. In many cases, this consisted of counselling with a CBT focus conducted for about $1 \mathrm{~h}$. Patients attended on average 8.2 (s.d. =6.4) faceto-face counselling sessions during the 12 week trial. A total of $25 \%$ of patients in this group received no recorded treatment.

\section{Statistical analyses}

Descriptive statistics (percentages) were calculated to describe participant characteristics at baseline, stratified by gender. Paired sample $t$-tests with Bonferroni corrections for multiple comparisons assessed within-group changes in depression and work capacity from baseline to 3-month follow-up. The overall effectiveness of the three interventions was assessed using analysis of covariance (ANCOVA) with baseline depression and work capacity scores entered as covariance factors in each model. This was considered necessary to control for the intercorrelation between baseline and follow-up scores that results when the same individuals complete a questionnaire twice. The strength of the intervention effect was assessed using the partial Eta-squared statistic (represented by the symbol $\eta^{2}$ ). Partial $\eta^{2}$ is the variance explained by a given variable after excluding the variance explained by other predictors. Effect sizes were interpreted using the following criteria: 0.01 , small; 0.06 , medium; and 0.14 or higher, a large effect size. ${ }^{25}$ Pairwise comparisons (independent sample $t$-tests) determined which group differences were statistically significant at 3 months post-treatment. Mean differences between groups with $95 \%$ confidence intervals are reported. For comparison purposes, a multilevel model was also performed, where the individual and time (baseline to 3 months) were the units of analysis. Possible age effects were examined by stratifying the data as follows: $18-34$ years $(n=283,30 \%)$, $35-49$ years $(n=346,36.7 \%)$ and $50-71$ years $(n=314,33.3 \%)$. Two patients had no recorded age. All analyses were performed using SPSS version 20.0.

\section{Results}

\section{Primary outcome}

Table 2 shows the change in mean depression (MADRS) scores at baseline and 3-month follow-up stratified by gender. Figure 2 illustrates these changes over time. Level of depression reduced significantly from baseline to post-treatment in all three treatment groups. There was a main effect of group with a small effect size, indicating that the improvements in depression at 3 months were significantly larger in both the physical exercise group (mean difference $2.99,95 \%$ CI $1.61-4.37, P<0.001$ ) and the ICBT group (mean difference $2.83,95 \%$ CI $1.47-4.19, P<0.001$ ), respectively. The treatment effect of exercise and ICBT was approximately equal. The main effect of gender fell slightly below statistical significance $(F(1,715)=3.43, P=0.06)$. When the data were stratified by age, results were comparable with the overall analysis (that is, favouring exercise and ICBT), with one partial exception. Among younger participants aged 18-34 years, the mean differences between treatment groups were not statistically significant $(F(2,207)=0.90, P=0.40)$. Mean depression scores at post-treatment in this age group were 10.4 (exercise group), 10.4 (ICBT group) and 11.1 (TAU group). Post-treatment group differences were statistically significant among adults aged $35-49$ years $(F(2,254)=6.58, \quad P<0.01)$ and $50-71$ years $(F(2,241)=5.17, \quad P<0.01)$. Multilevel modelling produced comparable outcomes with the ANCOVA analyses. A subgroup analysis was conducted to exclude patients taking antidepressants at baseline. The results indicated outcomes equivalent to the total sample analyses (data not shown).

\section{Secondary outcome}

Table 3 shows the change over time in self-rated work capacity scored on a single item scale ranging from 0 (no capacity) to 10 (highest possible capacity). Within-group improvements were 


\begin{tabular}{|c|c|c|c|c|c|c|}
\hline \multirow[b]{2}{*}{ Group } & \multicolumn{2}{|c|}{ Mean, s.d. } & \multirow{2}{*}{$\begin{array}{l}\text { Within-group } \\
\text { change, } t \text { (d.f.) }\end{array}$} & \multicolumn{2}{|c|}{ Main effect of treatment group, ANCOVA } & \multirow{2}{*}{$\begin{array}{c}\text { Effect size, } \\
\text { partial } \eta^{2}\end{array}$} \\
\hline & Baseline & 3 Months & & $F$ (d.f.) & $P$ & \\
\hline All participants & & & & $11.5(2,714)$ & $<0.001$ & 0.031 \\
\hline Treatment as usual & $20.9(7.5)$ & $13.8(8.9)$ & $12.6(226)^{*}$ & & & \\
\hline Physical exercise & $22.2(6.8)$ & $11.3(7.9)$ & $18.8(238)^{*}$ & & & \\
\hline Internet-based cognitive-behavioural therapy & $21.5(6.7)$ & $11.2(7.3)$ & $19.2(251)^{*}$ & & & \\
\hline Men & & & & $9.2(2,191)$ & $<0.001$ & 0.088 \\
\hline Treatment as usual & $21.5(8.1)$ & $16.9(10.6)$ & $4.1(49)^{\star}$ & & & \\
\hline Physical exercise & $21.9(6.7)$ & $10.8(8.2)$ & $10.3(75)^{*}$ & & & \\
\hline Internet-based cognitive-behavioural therapy & $22.2(5.4)$ & $12.7(8.1)$ & $9.3(68)^{*}$ & & & \\
\hline Women & & & & $5.6(2,519)$ & $<0.01$ & 0.021 \\
\hline Treatment as usual & $20.7(7.3)$ & $12.9(8.2)$ & $12.2(176)^{*}$ & & & \\
\hline Physical exercise & $22.4(6.9)$ & $11.6(7.7)$ & $15.6(162)^{*}$ & & & \\
\hline Internet-based cognitive-behavioural therapy & $21.3(7.1)$ & $10.6(6.9)$ & $16.8(182)^{*}$ & & & \\
\hline
\end{tabular}

(a)

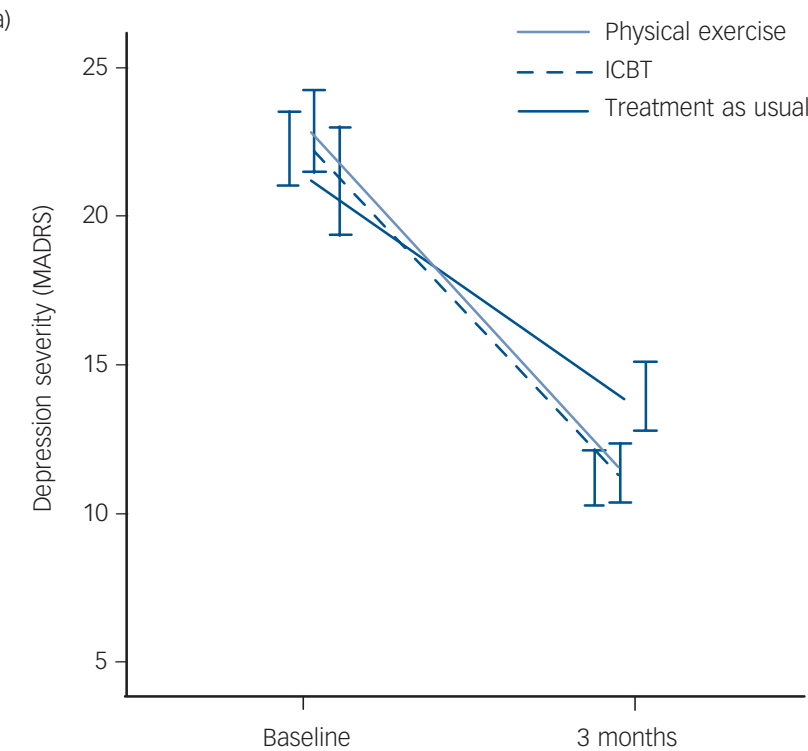

(b)

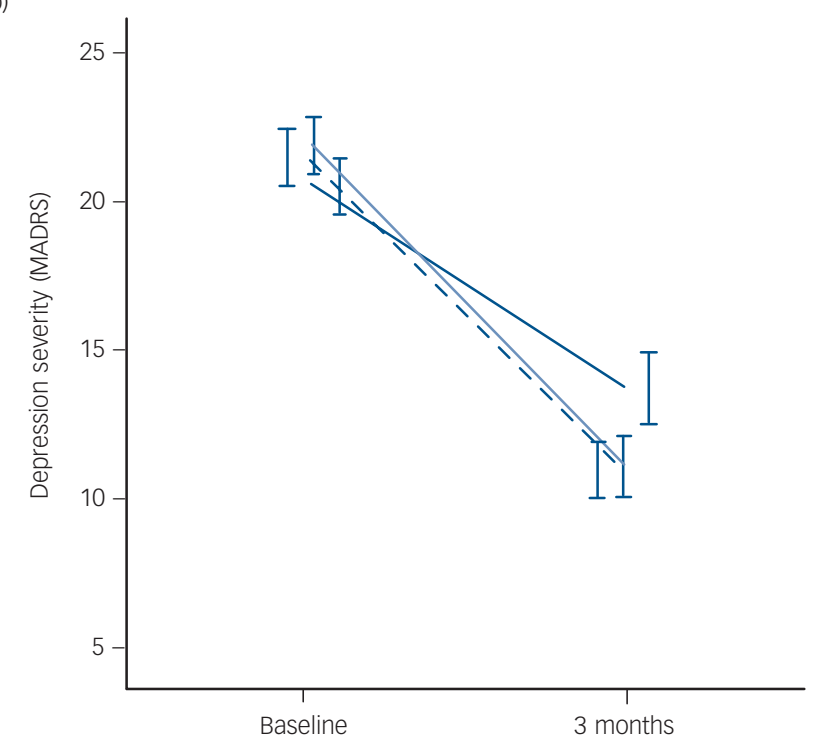

Fig. 2 Change over time in depression severity in (a) men and (b) women.

ICBT, internet-based cognitive-behavioural therapy; MADRS, Montgomery-Åsberg Depression Rating Scale. found in all three treatments, but there were no differences between the three groups at 3 months. A gender difference was found, with men rating greater improvements in work capacity over time compared with women, but the effect size was small.

\section{Response rates}

Of the 946 patients who completed a baseline assessment, 740 (78.2\%) participated in a post-treatment depression assessment. A smaller proportion of patients completed the work capacity survey $(67.6 \%)$. A drop-out analysis revealed no differences in response rates based on age, gender, depression status, work capacity or alcohol use at baseline. However, more people who dropped out were not working at baseline $(30 \%)$ compared with those who were employed $(23 \%, P<0.03)$ and were patients assigned to TAU $(14 \%)$ compared with ICBT $(9 \%, P<0.01)$ or physical exercise $(12 \%, P<0.02)$. The follow-up survey revealed that the majority of those who dropped out did so because of dissatisfaction with the randomisation outcome. Despite understanding that participation in the trial entailed a randomisation process, some patients were evidently disappointed when they did not receive their preferred treatment. A minority of patients withdrew because they felt the treatment regime was impractical and interfered with other activities. One patient relocated abroad and another could not be located.

Missing items on key variables ranged between $1 \%$ and $7 \%$ with one exception; $23 \%$ of items from the MADRS Question 2 (apparent sadness) were missing at follow-up. This occurred because Question 2 is based on an observational rating and about one-third of the MADRS follow-up interviews were conducted by telephone; consequently the question could not be rated. Otherwise, there were no discernible patterns associated with the missing data. As the first two items of the MADRS are highly correlated $(r=0.81)$ and logically related to each other, we used the last number carried forward (LNCF) method to impute these missing values. Separate analyses of the non-imputed MADRS data indicated almost identical results.

\section{Discussion}

\section{Key findings and interpretation}

In this study, all three interventions were associated with improvements in depressive symptoms at follow-up. However, the mean reduction in depression scores were significantly larger in the physical exercise and ICBT groups compared with TAU; an effect seen among both men and women. The overall treatment effect of 
Table 3 Within- and between-group treatment effects on work capacity ${ }^{a}$

\begin{tabular}{|c|c|c|c|c|c|c|}
\hline & \multicolumn{2}{|c|}{ Mean, s.d. } & \multirow{2}{*}{$\begin{array}{l}\text { Within-group } \\
\text { change, } t \text { (d.f.) }\end{array}$} & \multicolumn{2}{|c|}{ Main effect of treatment group, ANCOVA } & \multirow{2}{*}{$\begin{array}{l}\text { Effect size } \\
\text { partial } \eta^{2}\end{array}$} \\
\hline & Baseline & 3 Months & & $F$ (d.f.) & $P$ & \\
\hline Treatment group & & & & $0.93(2,639)$ & 0.39 & 0.003 \\
\hline Treatment as usual & $5.68(2.5)$ & $6.64(2.6)$ & $5.28(201)^{*}$ & & & \\
\hline Physical exercise & $5.58(2.4)$ & $6.69(2.6)$ & $6.34(209)^{*}$ & & & \\
\hline Internet-based cognitive-behavioural therapy & $5.52(2.5)$ & $6.84(2.4)$ & $8.16(230)^{*}$ & & & \\
\hline Gender & & & & $6.0(1,640)$ & $<0.05$ & 0.009 \\
\hline Men & $5.67(2.4)$ & $6.43(2.6)$ & $4.16(183)^{*}$ & & & \\
\hline Women & $5.56(2.5)$ & $6.85(2.5)$ & $10.92(485)^{\star}$ & & & \\
\hline
\end{tabular}

exercise and ICBT was approximately equal. An age effect was observed; adults over 34 years responded more favourably to the exercise and ICBT interventions compared with TAU. A similar trend was found among younger adults (18-34 years), but the group differences were not significant at post-treatment. Work capacity improved over time but there were no group differences.

The clinical relevance of the observed improvements in depression are important to consider. ${ }^{23}$ Snaith et al ${ }^{26}$ propose a cut-off score of 20 points for moderate depression (with 7-19 points for mild severity). Mean MADRS scores at baseline ranged between 20.7 and 22.4 points, indicating that most participants were experiencing a 'moderate' depression at the initial assessment. At post-treatment (3 months), mean MADRS scores were reduced significantly in all three treatment groups to a level corresponding with 'mild' depression severity. Also relevant, is that among men randomised to TAU, the MADRS score (16.9) remained within the moderate category at follow-up, suggesting that men were less responsive than women to the interventions offered in usual care. In sum, the improvements seen in all three treatment groups were clinically meaningful, but patients in the exercise and ICBT groups improved significantly more than patients receiving TAU.

In addition to negative mood states, depression is associated with numerous functional impairments. Recently, there have been calls to include functional outcomes in studies investigating treatment effects on depression. ${ }^{27}$ Perceived work capacity was assessed before and after treatment because it is an important indicator of a person's ability and willingness to work; a key functional outcome in this context. Self-rated work capacity improved equally in all three treatment groups at follow-up.

Our findings are broadly consistent with recent reviews supporting the mood-enhancing benefits of exercise on depression. A Cochrane review of 35 RCTs (1356 participants) comparing exercise with no treatment or a control intervention reported a pooled standardised mean difference of $-0.62(95 \%$ CI -0.81 to -0.42$)$, indicating a moderate clinical effect of exercise. ${ }^{28}$ When compared with psychological or pharmacological treatment (12 studies), exercise was found to be equally but not more effective than usual care. Results from our investigation are also comparable with several controlled studies. In a multicenter trial involving 2322 patients treated for heart failure with a mild to moderate depression, Blumenthal et al ${ }^{29}$ randomised participants to either 12 months of supervised/home-based aerobic exercise, or usual care for heart failure (with no planned exercise). Compared with usual care, aerobic exercise resulted in a modest but statistically significant reduction in mean depression scores at both 3- and 12-month follow-up. In an often cited study, Dunn et al explored dose-response relationships to exercise in 80 adults aged 20-45 years with mild to moderate depression. ${ }^{30}$ Participants were randomised to one of four supervised exercise treatment groups lasting 12 weeks, or a control condition. Of importance, the study showed that exercise by itself is effective in the treatment of depression, and that the amount of exercise needed is equivalent to consensus public health recommendations.

There is sound neurobiological and clinical trial evidence supporting the mental health benefits of exercise, yet some studies have failed to observe positive effects. In one of the largest trials of physical activity and depression conducted in primary care, Chalder et al randomised 351 adults aged 18-69 to either usual care plus a supported exercise intervention lasting 8 months, or to usual care alone. ${ }^{31}$ At 4-month follow-up (the primary outcome end-point) no differences in depression were observed between the physical activity and usual care groups. Moreover, there was no evidence of an intervention effect on mood at 8 and 12 months. There are notable similarities between the Chalder et al study and the present investigation; both were conducted in primary care settings and included a large number of adults voluntarily seeking treatment for depressive symptoms. However, there are also key differences that may explain the disparate results. In the present study, the aim was to supervise patients through a structured 12 -week physical exercise regime. All exercise sessions were delivered in a standardised group setting for the duration of the trial. Adherence was encouraged and monitored through weekly contact with the patient's assigned trainer; a person known to the participants. In the Chalder et al study, the goal was to support an overall increase in physical activity. Participants were encouraged to engage in a range of physical exercises, but they were not supervised through a structured exercise programme. These key differences are relevant because recent research suggest that supervision of aerobic activity is a key predictor of successful treatment outcomes in depression trials. ${ }^{32}$ Thus, it is possible that the higher level of support and monitoring offered to our patients encouraged more frequent exercise participation and, in turn, greater improvements in depression outcomes.

The other intervention assessed in this trial was internet-based CBT. A recent but growing body of evidence suggests that internet-based interventions can have beneficial effects on depression and several studies have reported outcomes comparable with those seen here. ${ }^{10}$ To assess the effectiveness of ICBT delivered within routine care, Hedman et al conducted a cohort study $(n=1203)$ at a psychiatric out-patient clinic in Sweden. ${ }^{33}$ The duration of the trial and the treatment delivery format were similar to the present one. However, treatment in the Hedman et al study focused solely on depression and was not individually tailored to address comorbid symptoms or work-related problems. As in the current trial, participants also had access to an online therapist who supervised progress, monitored suicidality and provided individual feedback on homework exercises (such as for behavioural activation). They 
found significant improvements in depression, and their effect size was somewhat smaller than in our study. Participants also reported improvements in sleep quality and reduced suicidal ideation; results that were maintained at 6-month follow-up. Recent ICBT studies have examined whether tailored $v$. standardised internet-based programmes have different effects on depression. In a RCT, Johansson et al found that both individually tailored and standardised ICBT were effective in reducing depressive symptoms and improving quality of life. ${ }^{34}$ However, a subgroup analysis revealed that the tailored version was more effective in treating patients with more severe depression. Recent reviews report a strong correlation between the degree of support provided in ICBT trials and better mental health outcomes. ${ }^{35}$ For this reason, regular monitoring and online support was an integral component of the our ICBT intervention.

Although internet-delivered health interventions offer a number of potential benefits (timely access, reduced health service costs, reaching stigmatised groups, etc), some studies have reported no benefits compared with TAU, ${ }^{36}$ and others have raised concerns about possible unintended consequences of internetdelivered mental health services. ${ }^{37}$ For example, it may be a poor substitute for face-to-face contact with a clinician in some instances. Providing an intervention via the internet for individuals living with a health problem they feel is stigmatised could also discourage the issue from being discussed beyond the relative anonymity of the internet, thus reinforcing the stigma. The findings presented in this study are promising, however, much of the supporting evidence for ICBT emanates from efficacy trials where the interventions have been delivered under optimal conditions. Studies of treatment effectiveness in 'real-world' settings remain scarce.

\section{Strengths and limitations}

To our knowledge, this is the largest effectiveness trial of physical exercise and ICBT for depression in primary care. The RCT design and the comparison of three treatment modalities in a single study are strengths of the investigation. The randomisation was performed remotely from where the RCT took place and participants were personally assessed in addition to making selfratings. Research nurses responsible for follow-up assessments were masked to treatment allocation. The inclusion of patients using medication is both a strength and a weakness; it makes the intervention less 'pure' but increases the external validity of the findings.

Several limitations are acknowledged. Patients in the TAU group received on average 8.2 face-to-face counselling sessions with their primary care physician, which was a lower average frequency of treatment compared with the exercise and ICBT interventions. Consequently, we cannot exclude the possibility that the favourable outcomes associated with these interventions were at least partly attributable to more frequent engagement in treatment. Importantly however, usual care in this study was not a control group equivalent; most patients were actively engaged in treatment. As in previous studies, our main focus has been on the resolution of depressive symptoms. Although this is clearly important, from a clinical perspective, it could be argued that quality of life is a more appropriate measure of functional improvement following depression treatment. Other measures, such as psychosocial functioning are also important to consider. The single item used to assess work capacity may have been inadequate; factors such as absenteeism (days off work) and level of productivity while at work are relevant indicators of work ability that were not assessed here. Another study limitation is that we do not know how many patients were invited to participate in the trial but declined. It was not feasible for the primary care centres involved in the study, nor the researchers, to collect these data. Finally, we acknowledge that adherence to the physical exercise and ICBT interventions was suboptimal, despite ongoing monitoring, which may have lowered the potential treatment effects of these interventions.

\section{Clinical implications and future research}

Prescribed physical exercise at a frequency of at least once per week and therapist supported, individually tailored ICBT are more effective short-term treatments for mild to moderate depression than general clinical management in primary healthcare. Despite strong evidence supporting the use of exercise to treat mental health problems, and the availability of guidelines for exercise prescription, ${ }^{38,39}$ physical activity is rarely used as a treatment for depression in Sweden. According to data published in 2012, only 1 out of 1000 visits to primary care physicians resulted in the prescription of exercise. ${ }^{40}$ The evidence supporting exercise for better mental health is substantial. We propose that physical activity should be more widely prescribed for depression in primary care settings. To date, over 1200 patients have received ICBT for depression through regular healthcare in Stockholm county. ${ }^{33}$ This mode of treatment is increasing in other parts of Sweden. Our results are consistent with several studies demonstrating positive effects of supported internet treatments for depression. Future investigations of dose-response are needed for both physical exercise and ICBT interventions. Age and gender effects should be examined closely, and greater attention should be given to assessing the unintended consequences of internet-based treatments for depression.

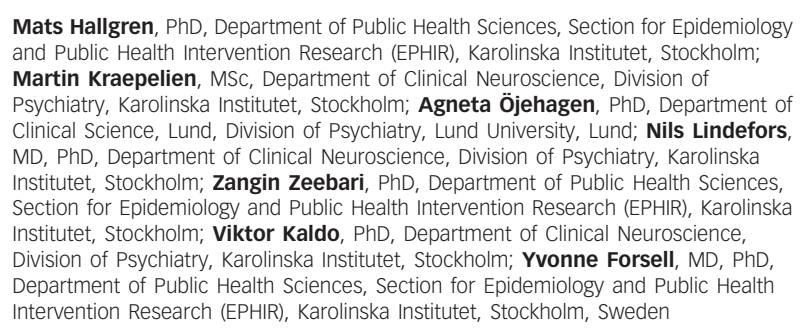

Mats Hallgren, PhD, Department of Public Health Sciences, Section for Epidemiology and Public Health Intervention Research (EPHIR), Karolinska Institutet, Stockholm; Martin Kraepelien, MSc, Department of Clinical Neuroscience, Division of Psychiatry, Karolinska Institutet, Stockholm; Agneta öjehagen, PhD, Department of Clinical Science, Lund, Division of Psychiatry, Lund University, Lund; Nils Lindefors, MD, PhD, Department of Clinical Neuroscience, Division of Psychiatry, Karolinska Institutet, Stockholm; Zangin Zeebari, PhD, Department of Public Health Sciences, Institutet, Stockholm; Zangin Zeebari, PhD, Department of Public Health Sciences,
section for Epidemiology and Public Health Intervention Research (EPHIR), Karolinska Institutet, Stockholm; Viktor Kaldo, PhD, Department of Clinical Neuroscience, Division of Psychiatry, Karolinska Institutet, Stockholm; Yvonne Forsell, MD, PhD, Department of Public Health Sciences, Section for Epidemiology and Public Health Intervention Research (EPHIR), Karolinska Institutet, Stockholm, Sweden

Correspondence: Mats Hallgren, Department of Public Health Sciences, Section for Epidemiology and Public Health Intervention Research (EPHIR), Karolinska Institutet, SE-171 77 Stockholm, Sweden. Email: mats.hallgren@ki.se

First received 6 Nov 2014, final revision 26 Mar 2015, accepted 29 Mar 2015

\section{Funding}

The Regassa project has been financed by the Stockholm county Council, Sweden. M.H. is currently funded by a post-doctoral stipend from the Brain Foundation, Sweden.

\section{References}

1 OECD. Mental health and work: Sweden. OECD, 2013.

2 Sobocki $\mathrm{P}$, Jonsson $\mathrm{B}$, Angst J, Rehnberg C. Cost of depression in Europe. J Ment Health Policy Econ 2006; 9: 87-98.

3 Forsell $\mathrm{Y}$. The pathway to meeting need for mental health services in Sweden. Psychiatr Serv 2006; 57: 114-9.

4 Rethorst CD, Wipfli BM, Landers DM. The antidepressive effects of exercise: a meta-analysis of randomized trials. Sports Med 2009; 39: 491-511.

5 Rimer J, Dwan K, Lawlor DA, Greig CA, McMurdo M, Morley W, et al. Exercise for depression. Cochrane Database Syst Rev 2012; 7: CD004366.

6 Dunn AL, Jewell JS. The effect of exercise on mental health. Curr Sports Med Rep 2010; 9: 202-7. 
7 Bjornebekk A, Mathe AA, Brene S. The antidepressant effect of running is associated with increased hippocampal cell proliferation. Int $J$ Neuropsychopharmacol 2005; 8: 357-68.

8 Eyre HA, Papps E, Baune BT. Treating depression and depression-like behavior with physical activity: an immune perspective. Front Psychiatry 2013; 4 : 3.

9 Barbour KA, Edenfield TM, Blumenthal JA. Exercise as a treatment for depression and other psychiatric disorders: a review. J Cardiopulm Rehabil Prev 2007; 27: 359-67.

10 Cuijpers $P$, van Straten A, Andersson G. Internet-administered cognitive behavior therapy for health problems: a systematic review. J Behav Med 2008; 31: 169-77.

11 Cuijpers $P$, van Straten A, Andersson G, van Oppen P. Psychotherapy for depression in adults: a meta-analysis of comparative outcome studies. J Consult Clin Psychol 2008; 76: 909-22.

12 Moritz S, Schilling L, Hauschildt M, Schroder J, Treszl A. A randomized controlled trial of internet-based therapy in depression. Behav Res Ther 2012; 50: $513-21$.

13 Wagner B, Horn AB, Maercker A. Internet-based versus face-to-face cognitive-behavioral intervention for depression: a randomized controlled non-inferiority trial. J Affect Disord 2014; 152-154: 113-21.

14 Andersson G, Cuijpers P, Carlbring P, Riper H, Hedman E. Guided Internetbased vs. face-to-face cognitive behavior therapy for psychiatric and somatic disorders: a systematic review and meta-analysis. World Psychiatry 2014; 13 288-95

15 Kroenke K, Spitzer RL, Williams JB. The PHQ-9: validity of a brief depression severity measure. J Gen Intern Med 2001; 16: 606-13.

16 SCB. Labor force survey, October 2011. Statistics Sweden, 2014

17 Sheehan DV, Lecrubier $\mathrm{Y}$, Sheehan $\mathrm{KH}$, Amorim $\mathrm{P}$, Janavs J, Weiller $\mathrm{E}$, et al. The Mini-International Neuropsychiatric Interview (M.I.N.I.): the developmen and validation of a structured diagnostic psychiatric interview for DSM-IV and ICD-10. J Clin Psychiatry 1998; 59 (suppl 20): 22-33; quiz 34-57.

18 Kallmen $\mathrm{H}$, Wennberg $\mathrm{P}$, Leifman $\mathrm{H}$, Bergman $\mathrm{H}$, Berman $\mathrm{AH}$. Alcohol habits in Sweden during 1997-2009 with particular focus on 2005 and 2009 , assessed with the AUDIT: a repeated cross-sectional study. Eur Addict Res 2011; 17: 90-6.

19 Saunders JB, Aasland OG, Babor TF, de la Fuente JR, Grant M. Developmen of the Alcohol Use Disorders Identification Test (AUDIT): WHO collaborative project on early detection of persons with harmful alcohol consumption-II. Addiction 1993; 88: 791-804.

20 American Psychiatric Association. Diagnostic and Statistical Manual of Mental Disorder (4th edn) (DSM-IV). APA, 1994

21 Rabin R, de Charro F. EQ-5D: a measure of health status from the EuroQo Group. Ann Med 2001; 33: 337-43.

22 Bergman $\mathrm{H}$, Kallmen $\mathrm{H}$. Alcohol use among swedes and a psychometric evaluation of the alcohol use disorders identification test. Alcohol Alcohol 2002; 37: 245-51.

23 Montgomery SA, Åsberg M. A new depression scale designed to be sensitive to change. Br J Psychiatry 1979; 134: 382-9.
24 Ahlstrom L, Grimby-Ekman A, Hagberg M, Dellve L. The Work Ability Index and single-item question: associations with sick leave, symptoms, and health-a prospective study of women on long-term sick leave. Scand J Work Environ Health 2010; 36: 404-12.

25 Richardson JTE. Eta squared and partial eta squared as measures of effect size in educational research. Educ Res Rev 2011; 6: 135-47.

26 Snaith RP, Harrop FM, Newby DA, Teale C. Grade scores of the MontgomeryAsberg Depression and the Clinical Anxiety Scales. Br J Psychiatry 1986; 148: 599-601.

27 Greer TL, Kurian BT, Trivedi $\mathrm{MH}$. Defining and measuring functional recovery from depression. CNS Drugs 2010; 24: 267-84.

28 Cooney GM, Dwan K, Greig CA, Lawlor DA, Rimer J, Waugh FR, et al. Exercise for depression. Cochrane Database Syst Rev 2013; 9: CD004366.

29 Blumenthal JA, Babyak MA, O'Connor C, Keteyian S, Landzberg J, Howlett J, et al. Effects of exercise training on depressive symptoms in patients with chronic heart failure: the HF-ACTION randomized trial. JAMA 2012; 308: 465-74

30 Dunn AL, Trivedi MH, Kampert JB, Clark CG, Chambliss HO. Exercise treatment for depression: efficacy and dose response. Am J Prev Med 2005; 28: $1-8$

31 Chalder M, Wiles NJ, Campbell J, Hollinghurst SP, Haase AM, Taylor AH, et al. Facilitated physical activity as a treatment for depressed adults: randomised controlled trial. BMJ 2012; 344: e2758.

32 Stanton R, Reaburn P. Exercise and the treatment of depression: a review of the exercise program variables. J Sci Med Sport 2014; 17: 177-82.

33 Hedman E, Ljotsson B, Kaldo V, Hesser $\mathrm{H}$, El Alaoui S, Kraepelien M, et al. Effectiveness of internet-based cognitive behaviour therapy for depression in routine psychiatric care. J Affect Disord 2014; 155: 49-58.

34 Johansson $R$, Sjoberg $E$, Sjogren $M$, Johnsson $E$, Carlbring $P$, Andersson $T$, et al. Tailored vs. standardized internet-based cognitive behavior therapy for depression and comorbid symptoms: a randomized controlled trial. PLOS One 2012; 7: e36905.

35 Johansson R, Andersson G. Internet-based psychological treatments for depression. Expert Rev Neurother 2012; 12: 861-9; quiz 870.

36 Kivi M, Eriksson MC, Hange D, Petersson EL, Vernmark K, Johansson B, et a Internet-based therapy for mild to moderate depression in swedish primary care: short term results from the PRIM-NET randomized controlled trial. Cogn Behav Ther 2014; 43: 289-98.

37 Griffiths F, Lindenmeyer A, Powell J, Lowe P, Thorogood M. Why are health care interventions delivered over the internet? A systematic review of the published literature. J Med Internet Res 2006; 8: e10.

38 Sundberg CJ, Jansson A, Edling C, Wadman M. Physical Activity in the Prevention and Treatment of Disease. Professional Associations for Physical Activity, 2010

39 Rethorst $\mathrm{CD}$, Trivedi $\mathrm{MH}$. Evidence-based recommendations for the prescription of exercise for major depressive disorder. J Psychiatr Pract 2013; 19: 204-12.

40 Kallings LV. Physical activity on prescription-an underutilized resource. Statistics on prescription shows large variations between counties [in Swedish]. Lakartidningen 2012; 109: 2348-50. 